\title{
Triple dose of gadolinium-DTPA and delayed MRI in patients with benign multiple sclerosis
}

\author{
M Filippi, R Capra, A Campi, B Colombo, F Prandini, N Marcianò, R Gasparotti, \\ G Comi
}

\begin{abstract}
Objectives-To evaluate whether a triple dose of gadolinium-DTPA (Gd-DTPA) or delayed MRI increase the number, size, and conspicuousness of enhancing lesions in patients with benign multiple sclerosis. Methods-T1 weighted brain MRI was carried out on 20 patients with benign multiple sclerosis (expanded disability status scale $<3$ with a disease duration $>10$ years) in two sessions. In the first session, one scan was obtained before and two scans five to seven minutes and 20-30 minutes after the injection of $0.1 \mathrm{mmol} / \mathrm{kg}$ Gd-DTPA (standard dose). In the second session, six to 24 hours later, the same procedure was repeated with $0.3 \mathrm{mmol} / \mathrm{kg}$ Gd-DTPA (triple dose).
\end{abstract}

Results-Nine enhancing lesions were found in seven patients (35\%) using the standard dose of Gd-DTPA. The numbers of enhancing lesions increased to $13(P=$ 0.03 ) and the number of patients with such lesions to eight $(40 \%)$ on the delayed standard dose scans. On the early triple dose scans, we found 19 enhancing lesions in 10 patients $(50 \%)$. The number of enhancing lesions was significantly higher $(P=0.01)$ than that obtained with the early standard dose. The number of enhancing lesions was 18 and the number of "active" patients $11(55 \%)$ on the delayed triple dose scans. The enhancing areas increased progressively from the early standard dose scans to the delayed triple dose scans. The contrast ratios of the lesions detected in the early standard dose scans was lower than those of lesions present in the early $(P=0.01)$ and delayed $(P=0.04)$ triple dose scans.

Conclusions-More enhancing lesions were detected in patients with benign multiple sclerosis with both delay of MRI and the use of a triple dose of Gd-DTPA suggesting that the amount of inflammation in the lesions of such patients is mild and heterogeneous.

(F Neurol Neurosurg Psychiatry 1996;60:526-530)

Keywords: benign multiple sclerosis; magnetic resonance imaging; delayed MRI; triple dose gadoliniumDTPA

Patients with benign multiple sclerosis are characterised by a low frequency of clinical relapses and by a mild extent of $\mathrm{T} 2$ weighted abnormalities in the brain..$^{12}$ In addition, longitudinal MRI studies using the standard dose of gadolinium-DTPA (Gd-DTPA) ${ }^{34}$ showed that patients with benign multiple sclerosis also have a much lower rate of activity compared with early relapsing-remitting multiple sclerosis. However, we do not know whether this means that in benign multiple sclerosis there are fewer enhancing lesions or the "active" lesions just have lower degrees of blood-brain barrier damage and inflammation. The latter might be detected using a triple dose of Gd-DTPA or delayed scanning, as already shown for patients with primary progressive multiple sclerosis, ${ }^{5}$ in whom lesions are characterised by moderate inflammation. ${ }^{6}$ In lesions in which the damage to the bloodbrain barrier is less severe and with consequently decreased blood-brain barrier permeability constants, ${ }^{78}$ it is indeed conceivable that higher blood concentrations of the Gd-DTPA or more time for the contrast material to leak into the lesions are needed to detect enhancement.

This study had three aims: (1) to evaluate whether the delayed scanning after the injection of the standard dose or the triple dose of Gd-DTPA might increase the number, the area, and the conspicuousness of enhancing lesions in patients with benign multiple sclerosis; (2) to evaluate whether combined use of the triple dose of Gd-DTPA and delayed scanning might result in further improvement of enhanced MRI sensitivity; and (3) to obtain new information about the nature of enhancing lesions in this clinical subgroup of the disease.

\section{Patients and methods}

PATIENTS

Patients were recruited from the multiple sclerosis population attending the multiple sclerosis center of the Scientific Institute Ospedale San Raffaele, University of Milan and the Department of Neurology, Spedali Civili, University of Brescia, Italy. Patients with benign disease were defined as those having a Kurtzke expanded disability status scale (EDSS) ${ }^{9}$ score of 3 or less after a disease duration of greater than 10 years. All patients had CSF oligoclonal bands. Patients were only included if they had had no clinical relapses and had not had immunosuppressive or immunomodulating treatments (including steroids) for at least six months. At the time MRI was performed, each patient was assessed neurologically and disability was 
assessed by the EDSS. Written informed consent was obtained from all the patients before inclusion in the study.

\section{MRI}

Brain MRI was performed in both the centres with identical machines (Siemens Magnetom SP63, 1.5 Tesla field strength). T1 weighted images (SE 768/14, $5 \mathrm{~mm}$ contiguous axial slices, $256 \times 256$ image matrix, field of view $=230 \mathrm{~mm}$ ) were obtained six times in two sessions separated by a period of 24 hours. In the first session, the $\mathrm{T} 1$ weighted scans were obtained before and five to seven minutes and 20 to 30 minutes after the injection of the standard dose $(0.1 \mathrm{mmol} / \mathrm{kg})$ of Gd-DTPA (Schering, Berlin, Germany). On the same occasion, before the injection of Gd-DTPA, moderately T2 weighted MRI of the brain was also performed (SE 2000/50, $5 \mathrm{~mm}$ contiguous axial slices, $256 \times 256$ image matrix, field of view $=230 \mathrm{~mm}$ ). In the second session, the same procedure was repeated (except for the acquisition of the T2 weighted images) after the injection of the triple dose $(0.3 \mathrm{mmol} / \mathrm{kg})$ of Gd-DTPA. Patients were repositioned according to published guidelines. ${ }^{10}$

Image review of enhanced $\mathrm{T} 1$ weighted scans in terms of the numbers of enhancing lesions was performed in three stages, always considering the abnormalities visible on T2 weighted and on unenhanced $\mathrm{T} 1$ weighted scans:

\section{Stage 1}

Each of the six sets of T1 weighted images from each patient was reviewed independently by two of us (MF and $\mathrm{RC}$ ), unaware of the Gd-DTPA dose used and the patient to whom the scans belonged. Each lesion was scored according to size (small: diameter $\leqslant 5 \mathrm{~mm}$; intermediate: diameter $=6-10 \mathrm{~mm}$; large: diameter > $10 \mathrm{~mm}$ );

\section{Stage 2}

Next, the six sets of images were reviewed together (again without knowing the GdDTPA doses used) and each lesion was reassessed by agreement;

\section{Stage 3}

Finally, the six sets of images were compared and each lesion once again reassessed by

Enhancing lesions detected in the four conditions studied *

\begin{tabular}{lllll}
\hline $\begin{array}{l}\text { Patient } \\
\text { No }\end{array}$ & $\begin{array}{l}\text { Early standard } \\
\text { dose scan }\end{array}$ & $\begin{array}{l}\text { Delayed standard } \\
\text { dose scan }\end{array}$ & $\begin{array}{l}\text { Early triple } \\
\text { dose scan }\end{array}$ & $\begin{array}{l}\text { Delayed triple } \\
\text { dose scan }\end{array}$ \\
\hline 1 & 0 & 0 & 0 & $\mathrm{a}(\mathrm{s})$ \\
2 & $\mathrm{a}(\mathrm{s})$ & $\mathrm{a}(\mathrm{s}) ; \mathrm{b}(\mathrm{l}) \dagger$ & $\mathrm{a}(\mathrm{s}) ; \mathrm{b}(\mathrm{l}) \dagger$ & $\mathrm{a}(\mathrm{s}) ; \mathrm{b}(\mathrm{l}) \dagger$ \\
3 & $\mathrm{a}(\mathrm{i})$ & $\mathrm{a}(\mathrm{i})$ & $\mathrm{a}(\mathrm{i})$ & $\mathrm{a}(\mathrm{i})$ \\
4 & $\mathrm{a}(\mathrm{i}) ; \mathrm{b}(\mathrm{i}) ; \mathrm{c}(\mathrm{s})$ & $\mathrm{a}(\mathrm{i}) ; \mathrm{b}(\mathrm{i}) ; \mathrm{c}(\mathrm{s}) ; \mathrm{d}(\mathrm{s})$ & $\mathrm{a}(\mathrm{i}) ; \mathrm{b}(\mathrm{i}) ; \mathrm{c}(\mathrm{s}) ; \mathrm{d}(\mathrm{s})$ & $\mathrm{a}(\mathrm{i}) ; \mathrm{b}(\mathrm{i}) ; \mathrm{c}(\mathrm{s}) ; \mathrm{d}(\mathrm{s})$ \\
5 & $\mathrm{a}(\mathrm{s})$ & $\mathrm{a}(\mathrm{s})$ & $\mathrm{a}(\mathrm{s})$ & $\mathrm{a}(\mathrm{s})$ \\
6 & $\mathrm{a}(\mathrm{s})$ & $\mathrm{a}(\mathrm{i})$ & $\mathrm{a}(\mathrm{i}) ; \mathrm{b}(\mathrm{s}) ; \mathrm{c}(\mathrm{i})$ & $\mathrm{a}(\mathrm{l}) ; \mathrm{b}(\mathrm{s})$ \\
7 & $\mathrm{a}(\mathrm{i})$ & $\mathrm{a}(\mathrm{i})$ & $\mathrm{a}(\mathrm{l}) ; \mathrm{b}(\mathrm{s}) ; \mathrm{c}(\mathrm{s})$ & $\mathrm{a}(\mathrm{l}) ; \mathrm{b}(\mathrm{s}) ; \mathrm{c}(\mathrm{s})$ \\
8 & 0 & $\mathrm{a}(\mathrm{s})$ & $\mathrm{a}(\mathrm{i})$ & $\mathrm{a}(\mathrm{i})$ \\
9 & 0 & $0(\mathrm{i})$ & $\mathrm{a}(\mathrm{i})$ \\
10 & $\mathrm{a}(\mathrm{i})$ & $\mathrm{a}(\mathrm{l})$ & $\mathrm{a}(\mathrm{l}) ; \mathrm{b}(\mathrm{s})$ & $\mathrm{a}(\mathrm{l})$ \\
11 & 0 & $\mathrm{a}(\mathrm{s})$ & $\mathrm{a}(\mathrm{s})$ & $\mathrm{a}(\mathrm{s})$ \\
\hline
\end{tabular}

^Patients with no enhancing lesions in all the four conditions are not reported. The same lesions seen in the different conditions are designed with the same letters. The size of each lesion is reported in parenthesis: $\mathrm{s}=$ small; $\mathrm{i}=$ intermediate; $1=$ large. In this lesion the enhancement was ring-like. agreement. The values obtained at the end of this review process entered statistical analysis.

The areas and the contrast ratios for all the regions which were considered enhancing lesions at the end of this review were measured by a single rater (MF), using a mouse controlled cursor on the computer display. The contrast ratio was calculated by dividing the mean signal intensity of each lesion by the mean signal intensity (weighted for the number of pixels) obtained for two areas in the CSF. The areas in the CSF chosen were usually in the same slices in which the lesions were present. When this was not possible, the slices closest to the lesions in which CSF was present were considered.

Lesion volumes present on $\mathrm{T} 2$ weighted scans were measured by one of us (MF) using a semiautomated thresholding technique. ${ }^{11} 12$

\section{STATISTICAL ANALYSIS}

The differences in the clinical variables, the total lesion load on T2 weighted images, and the percentage increase in the number of enhancing lesions detected with the triple dose compared with the standard dose between patients recruited in the two centres were analysed by two tailed Student's $t$ test for nonpaired data when the data were normally distributed and by the Mann-Whitney $U$ test when they were not. The relation between the percentage increase in the number of enhancing lesions detected with the triple dose compared with the standard dose and the intervals between the two doses was tested with the Spearman rank correlation coefficient. The differences between the four conditions in number and total area per patient of enhancing lesions (only for patients with at least one enhancing lesion) were analysed by the Wilcoxon signed rank test. The differences in contrast ratio between enhancing lesions detected in the four conditions were studied by one way analysis of variance (ANOVA) (post hoc analysis: two tailed Student's $t$ test for unpaired data).

\section{Results}

CLINICAL DATA

Twenty patients entered the study. Ten patients were recruited in Milan and 10 in Brescia. Their mean age (SD) was $41.4(6.9)$ years, mean duration of the disease (SD) was $15 \cdot 1(4 \cdot 3)$ years, and median EDSS score was 1 with a range of $0-3$. There were no statistical differences in these clinical characteristics for patients recruited in the two centres.

\section{MRI}

The median lesion load on T2 weighted images was $8990 \mathrm{~mm}^{3}$ with a range of 3440-65 $840 \mathrm{~mm}^{3}$. There were no statistically significant differences in total lesion load between the two centres.

The table gives the number and size of enhancing lesions detected in each patient with the four conditions. There were nine enhancing lesions in seven patients $(35 \%)$ on the early standard dose scans. The number of 
(A) Axial T1 weighted (SE 768/14) image obtained five to seven minutes after the injection of the standard dose $(0.1 \mathrm{mmol} / \mathrm{kg})$ of gadolinium-DTPA. No enhancing lesions are visible. (B) Axial T1 weighted (SE 768/14) image obtained five to seven minutes after the injection of the triple dose $(0.3 \mathrm{mmol} / \mathrm{kg})$ of gadolinium-DTPA. An enhancing lesion is visible in the right subcortical white matter of the occipital lobe
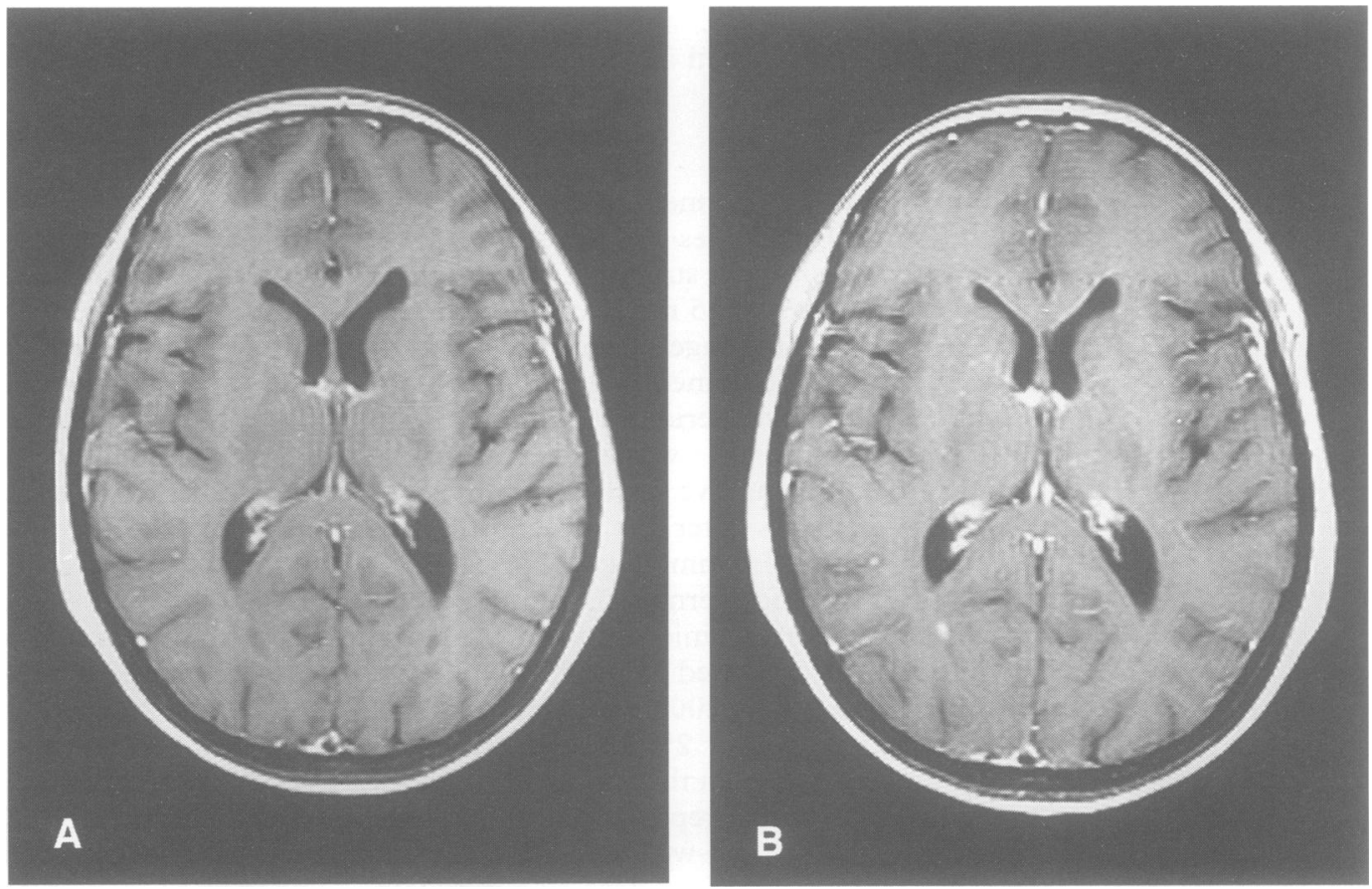

enhancing lesions increased to $13(P=0.03)$ and the number of patients with an active scan to nine $(45 \%)$ on the delayed standard dose scans. We found two enhancing lesions in two patients with no enhancing lesions on the standard dose scans. The other two lesions were found in patients who already had enhancing lesions. Another two enhancing lesions increased in size.

No enhancing lesions were found on the scans obtained before the injection of the triple dose of Gd-DTPA. Using the triple dose, the number of enhancing lesions increased to 19 (figure) and the number of "active" scans to $10(50 \%)$ on the early scans. The enhancing lesions detected on early triple dose scans were significantly more than those present on early standard dose scans $(P=0.01)$. A patient with no enhancing lesions on both the standard dose scans had one lesion on the early triple dose scans. The remaining five enhancing lesions were found in patients who had "active" lesions on the standard dose scans. Two other lesions increased in size when compared with those present on the delayed standard dose scan. The percentage increase in enhancing lesions between the standard dose and the triple dose of Gd-DTPA was not different between the patients recruited in the two centres and was not related to the interval between these two scans. On the delayed triple dose scans, we found 18 enhancing lesions in 11 patients $(55 \%)$. The number of enhancing lesions seen on these scans was significantly higher than that of early standard dose scans $(P=0.01)$. A patient with no enhancing lesions in all the previous scans had one small enhancing lesion on the delayed triple dose scan. On the other hand, two lesions present on the early triple dose scans were no longer detectable. One lesion increased from intermediate on early triple dose scans to large on delayed triple dose scans.

For the patients who had at least one enhancing lesion in one of the four conditions, the median enhancing lesion area per patient was 6.5 (range $0-69.4$ ) $\mathrm{mm}^{2}$ on the early standard dose, 14.9 (range $0-78.4$ ) $\mathrm{mm}^{2}$ on the delayed standard dose, $36 \cdot 1$ (range $0-82 \cdot 2$ ) $\mathrm{mm}^{2}$ on the early triple dose, and 37.5 (range $4 \cdot 7-88) \mathrm{mm}^{2}$ on the delayed triple dose scans. All the comparisons were significant (early $v$ delayed standard dose: $P=0.009$; early standard dose $v$ early triple dose: $\mathrm{P}=0.006$; early standard dose $v$ delayed triple dose: $\mathbf{P}=$ 0.004 ; delayed standard dose $v$ early triple dose: $\mathrm{P}=0.04$; delayed standard dose $v$ delayed triple dose: $P=0.005$ ) except for the comparison between early $v$ delayed triple dose, in which only a trend was found $(P=$ 0.07 ).

The mean (SD) contrast ratios were 2.9 $(0.4)$ for enhancing lesions detected on the early standard dose scans, $3.2(0.9)$ for those present in the delayed standard dose scans, 3.9 $(1 \cdot 1)$ for those present in the early triple dose scans, and $3.9(1.4)$ for those present in the delayed triple dose scans $(P=0.04)$. The contrast ratios of the lesions detected in the early standard dose scans were lower than those of lesions present in the early $(P=0.01)$ and delayed $(P=0.04)$ triple dose scans.

No patient complained of discomfort or presented side effects after the injection of the triple dose of Gd-DTPA.

\section{Discussion}

This study indicates that both the triple dose of Gd-DTPA and the delayed scanning can be used to increase MRI sensitivity in detecting enhancing lesions in patients with benign multiple sclerosis. However, it is important to note that nine of the 20 patients studied still had no gadolinium enhancement despite the use of triple dose scans and delayed scans, thus confirming that benign multiple sclerosis is a less inflammatory disease compared with other 
clinical subgroups of multiple sclerosis. ${ }^{4}$ The increased enhanced MRI sensitivity when a triple dose of Gd-DTPA and delayed scanning are used might depend on the heterogeneous nature of enhancing lesions in these patients, as a result of the time disseminated activity of the disease. Low permeability lesions and lesions with large leakage space ${ }^{78}$ clearly have visible enhancement only when higher blood concentrations of the contrast material are reached or longer time is given to the contrast material to leak within lesions with a damaged blood-brain barrier, as previously shown using delayed high dose CT. ${ }^{13-15}$ Previous longitudinal studies with conventional enhanced MRI found that in patients with benign multiple sclerosis the occurrence of enhancement is less frequent than in patients with early relapsingremitting multiple sclerosis. ${ }^{34}$ This difference might be explained by two situations, which are not mutually exclusive: (1) patients with benign multiple sclerosis have fewer "active" lesions in which blood-brain barrier dysfunction and inflammation have occurred or (2) patients with benign multiple sclerosis have higher proportions of lesions which, because of their pathological characteristics, go undetected with conventional MRI. The results of the present study suggest that both these explanations are valid. In a previous study ${ }^{16}$ with a triple dose of Gd-DTPA in 22 patients with relapsing-remitting and secondary progressive multiple sclerosis we found 138 enhancing lesions, 18 "active" scans and an enhancing area of $190.7 \mathrm{~mm}^{2}$, whereas we found only 19 lesions, 10 "active" scans and an enhancing area of $36.1 \mathrm{~mm}^{2}$ in these 20 patients with benign multiple sclerosis. Nevertheless, the number of enhancing lesions detected increased by $44 \%$ on standard dose delayed scans and by about $100 \%$ on the early triple dose scans, indicating that also in benign multiple sclerosis the pathological nature of enhancing lesions is heterogeneous with a large proportion of lesions characterised by low permeability to the contrast material or larger leakage space.

The low blood-brain barrier permeability of enhancing lesions in benign multiple sclerosis could be due to two factors. Firstly, these lesions might be in an advanced phase of their natural history, in which inflammation is partially subsided and some repair of the damaged blood-brain barrier has already occurred. ${ }^{7}$ Secondly, enhancing lesions in benign multiple sclerosis, for reasons that remain unknown, might be characterised by less severe inflammation and damage to the blood-brain barrier, regardless of their age. A previous pathological study ${ }^{6}$ has indicated that different degrees of inflammation are present in patients with multiple sclerosis with different courses of the disease. However, the role of these two factors can be clarified only by longitudinal studies with monthly MRI and triple dose of Gd-DTPA.

Leakage spaces of different sizes might also be responsible for the heterogeneous patterns of enhancement, as it would take longer or need more contrast material to fill larger leak- age spaces. The pathological substrates of an enlarged leakage space could be either oedema or tissue destruction secondary to severe demyelination and axonal loss. This second possibility seems extremely unlikely in patients with mild disability, as previous studies ${ }^{17-21}$ found MR markers of tissue destruction to be abnormal typically in severe disabled patients. However, this possibility cannot be ruled out completely, as we found a very large ring enhancing lesion only when delayed scanning was obtained or triple dose given. Ring enhancement is considered the hallmark of reactivation of chronic lesions, ${ }^{22}$ in which the hypointense centre could be the result of severe tissue disorganisation. ${ }^{20}$ The second possibility (perilesional oedema possibly due to blood-brain barrier damage and inflammation occurring at the edge of lesions) seems more convincing not only for the favourable course of the disease of the patients studied, but also for the fact that all the other enhancing lesions which were not present on conventional enhanced MRI were small or intermediate in size. This is also supported by the demonstration of enlargement of pre-existing lesions on both the delayed scans. In this respect, it is important to note that the enhancing area increased on triple dose delayed scans compared with early triple dose scans despite the fact that the number of lesions decreased, indicating a progressive diffusion of contrast material into the enlarged extracellular space secondary to oedema extending beyond the region with active breakdown of the blood-brain barrier.

A triple dose of Gd-DTPA enabled us to detect more enhancing lesions that were more conspicuous and had a larger enhancing area than just delaying the scans after the injection of the standard dose. It is possible that some of the lesions which enhanced on the triple dose scans would have enhanced also after the standard dose if the delayed scans were obtained after longer periods - that is, one hour or more. Kermode et $a l$ found that most enhancing lesions reached their maximum signal intensities around a mean of 29 minutes, but some lesions remained brighter even five hours after the injection. However, these latter lesions were typically large and presented ring enhancement, whereas smaller and homogeneously enhancing lesions had disappeared by 80 minutes. All the lesions we found on the triple dose scans which went undetected on the delayed standard dose scans were small or intermediate in size and had homogeneous enhancement. Therefore, this suggests that there are lesions in benign multiple sclerosis which for their pathological characteristics (low permeability and small leakage space) enhance only when high transmembrane concentrations of Gd-DTPA are reached.

The same considerations also apply for delaying the scans after the injection of the triple dose. We found only one lesion which did not enhance on the early triple dose scans and did so on the delayed triple dose scans and we think that it is unlikely this situation would have happened more often if the scan- 
ning delay had been increased. On the contrary, we found two enhancing lesions on early triple dose scans which were not seen when the delayed scan was obtained, thus indicating that longer delays after triple dose injection may lead to reduced sensitivity of enhanced MRI.

Our findings have diagnostic and therapeutic implications. The demonstration of lesions with different enhancing patterns could have an important impact for patients, not infrequent in clinical practice, with no or only mild neurological manifestations in whom aspecific abnormalities on T2 weighted scans can be present. ${ }^{23}$ Although not yet proved, it is indeed likely that enhancing lesions are very rare in small vessel disease associated with aging even when high doses of Gd-DTPA are used.

As regards treatment, interferon- $\beta$ is effective in inhibiting the opening of the blood-brain barrier in patients with relapsingremitting multiple sclerosis. ${ }^{24}$ It is also known that the amount of enhancement predicts the evolution of multiple sclerosis ${ }^{25}$ and that in about $20 \%$ of the patients, benign multiple sclerosis evolves to secondary progressive multiple sclerosis between 10 to 15 years after the onset. ${ }^{1}$ Longitudinal studies using the triple dose of Gd-DTPA might, therefore, be useful to identify patients with benign multiple sclerosis at higher risks of evolution who might benefit from treatment.

The program for lesion volume measurements was written by Drs D A G Wicks, P S Tofts, G J Barker, and M A Horsfield in the NMR Research Group, Institute of Neurology, Queen Square, London, UK, with generous financial assistance from the Multiple Sclerosis Society of Great Britain and Northern Ireland. The Dispimage display program was written and provided by $\mathrm{Mr} \mathrm{D}$ Plummer (Department of Medical Physics, University College, London, UK). We also thank Mr C Pereira
(Department of Neuroradiology, Scientific Institute Ospedale San Raffaele) for his skilful technical assistance in performing MRI.

1 Weinshenker BG. The natural history of multiple sclerosis. Neurol Clin 1995;13:119-46.

2 Thompson AJ, Kermode AG, MacManus DG, et al. Patterns of disease activity in multiple sclerosis: clinical and magnetic resonance imaging study. $B M F$ 1990;300: $631-4$.

3 Thompson AJ, Miller D, Youl B, et al. Serial gadoliniumenhanced MRI in relapsing-remitting multiple sclerosis of varying disease duration. Neurology 1992;42:60-3.

4 Kidd D, Thompson AJ, Kendall BE, Miller DH, McDonald WI. Benign form of multiple sclerosis: MRI evidence for less frequent and less inflammatory disease evidence for less frequent and less inflammatory disease

5 Filippi M, Campi A, Martinelli V, et al. Comparison of triple dose versus standard dose gadolinium-DTPA for detection of MRI enhancing lesions in patients with primary progressive multiple sclerosis. 7 Neurol Neurosurg Psychiatry 1995;59:540-4.

6 Revesz T, Kidd D, Thompson AJ, Barnard RO, McDonald WI. A comparison of the pathology of primary and secondary prot

7 Kermode AG, Tofts PS, Thompson AJ, et al. Heterogeneity of blood-brain barrier changes in multiple sclerosis: an MRI study with gadolinium-DTPA enhancement. Neurology 1990;40:229-35.

8 Tofts PS, Kermode AG. Measurement of the blood-brain Tofts PS, Kermode AG. Measurement of the blood-brain
barrier permeability and leakage space using dynamic MR imaging. Fundamental concepts. Magn Reson Med 1991;17:357-67.

9 Kurtzke JF. Rating neurologic impairment in multiple sclerosis: an expanded disability status scale (EDSS). Neurology 1983;33:1444-52.

10 Miller DH, Barkhof F, Berry I, Kappos L, Scotti G, Thompson AJ. Magnetic resonance imaging in monitoring the treatment of multiple sclerosis: concerted action guidelines. I Neurol Neurosurg Psychiatry 1991;54:683-8.

11 Wicks DAG, Tofts PS, Miller DH, et al. Volume measurements of multiple sclerosis lesions with magnetic resonance images: a preliminary study. Neuroradiology nance images:

12 Filippi M, Horsfield MA, Morrissey SP, et al. Quantitative brain MRI lesion load predicts the course of clinically isolated syndromes suggestive of multiple sclerosis. Neurology 1994;44:635-41.

13 Vinuela FV, Fox AJ, Debrun GM, Feasby TE, Ebers GC. New perspectives in computed tomography of multiple sclerosis. AfNR Am $\mathcal{F}$ Neuroradiol 1982;3:277-81.

14 Sears S, McCammon A, Bigelow R, Hayman LA. Maximizing the harvest of contrast enhancing lesions in multiple sclerosis. Neurology 1982;32:815-20.

15 Spiegel SM, Vinuela F, Fox AJ, Pelz DM. CT of multiple sclerosis: reassessment of delayed scanning with high doses of contrast material. $A \mathscr{F} \mathrm{Am} \mathcal{F}$ Roentgenol 1985;145:497-500.

16 Filippi M, Yousry T, Campi A, et al. Comparison of triple dose versus standard dose gadolinium-DTPA for detection of MRI enhancing lesions in patients with multiple sclerosis. Neurology 1996 (in press).

17 Filippi M, Barker GJ, Horsfield MA, et al. A quantitative brain MRI study of benign and secondary progressive multiple sclerosis. $\mathcal{F}$ Neurol 1994;241:246-51.

18 Gass A, Barker GJ, Kidd D, et al. Correlation of magnetisation transfer ratio with clinical disability in multiple sclerosis. Ann Neurol 1994;36:62-7.

19 Filippi M, Campi A, Dousset V, et al. A magnetization transfer imaging study of normal-appearing white matter in multiple sclerosis. Neurology 1995;45:478-82.

20 van Walderveen MAA, Barkhof F, Hommes OR, et al. Correlating MR imaging and clinical disease activity in multiple sclerosis: relevance of hypointense lesions on multiple sclerosis: re " $\mathrm{T}_{1}$-weighted") spin-echo images. short TR/short TE ("T, ${ }_{1}$-we
Neurology 1995;45:1684-90.

21 Kidd D, Thorpe JW, Thompson AJ, et al. Spinal cord MRI using multi-array coils and fast spin echo. II. Findings in multiple sclerosis. Neurology 1993;43:2632-7.

22 McDonald WI, Miller DH, Barnes D. The pathological evolution of multiple sclerosis. Neuropathol Appl Neurobiol 1992;18:319-34

23 Gerard G, Weisberg LA. MRI periventricular lesions in adults. Neurology 1986;36:998-1001.

24 Stone LA, Frank JA, Albert PS, et al. The effect of interferon-beta on blood-brain barrier disruptions demonstrated by contrast-enhanced magnetic resonance imaging in relapsing-remitting multiple sclerosis. Ann Neurol 1995;37:611-9.

25 Smith ME, Stone LA, Albert PS, et al. Clinical worsening in multiple sclerosis is associated with increased frequency and area of gadopentetate dimeglumine-enhancquency and area of gadopentetate dimeglumine-enhancing magnetic r. 